\title{
State of the art review
}

\section{Measuring lung function in airways diseases: current and emerging techniques}

Nayia Petousi,,$^{1,2^{*}}$ Nick P Talbot, ${ }^{1,2^{*}}$ Ian D Pavord ${ }^{1,2}$ and Peter A Robbins ${ }^{1,3}$

1. Oxford NIHR Respiratory Biomedical Research Centre, University of Oxford, Oxford, UK

2. Nuffield Department of Medicine, University of Oxford, Oxford, UK

3. Department of Physiology, Anatomy and Genetics, University of Oxford, Oxford, UK

*equal contribution

Corresponding author:

Nayia Petousi

Corresponding author's address:

NDM Research Building, Old Road Campus,

Oxford, OX3 7FZ

Corresponding author's tel:

$(+44) 7834632500$

Corresponding author's email:

nayia.petousi@dpag.ox.ac.uk

Word count: 4380 


\section{ABSTRACT}

Introduction. Chronic airways diseases, including asthma, chronic obstructive pulmonary disease (COPD) and cystic fibrosis, cause significant morbidity and mortality and are associated with high healthcare expenditure, in the UK and worldwide. For patients with these conditions, improvements in clinical outcomes are likely to depend upon the application of precision medicine, i.e. the matching of the right treatment to the right patient at the right time. In this context, the identification and targeting of 'treatable traits' is an important priority in airways disease, both to ensure the appropriate use of existing treatments, and to facilitate the development of new disease-modifying therapy. This requires better understanding of airway pathophysiology, but also an enhanced ability to make physiological measurements of disease activity and lung function and, if we are to impact on the natural history of these diseases, reliable measures in early disease.

Discussion. In this article, we outline some of the key challenges faced by the respiratory community in the management of airways disease, including early diagnosis, disease stratification, and monitoring of therapeutic response. In this context, we review the advantages and limitations of routine physiological measurements of respiratory function including spirometry, body plethysmography and diffusing capacity, and discuss less widely-used methods such as forced oscillometry, inert gas washout and the multiple inert gas elimination technique (MIGET). Finally, we highlight emerging technologies including imaging methods such as quantitative computed tomography and hyperpolarized gas magnetic resonance imaging as well as quantification of lung inhomogeneity using precise in-airway gas analysis and mathematical modeling.

Conclusion. These emerging techniques have the potential to enhance existing measures in the assessment of airways diseases, may be particularly valuable in early disease, and should facilitate the efforts to deliver precision respiratory medicine. 
KEYWORDS: Lung function, spirometry, inhomogeneity, airways disease, physiology, thoracic imaging

\section{INTRODUCTION}

Chronic obstructive airways disease (COPD) affects $\sim 200$ million people worldwide, ${ }^{1}$ is responsible for $>10 \%$ of acute NHS hospital admissions ${ }^{2}$ and will become the third-leading cause of death by 2020. ${ }^{1}$ Asthma affects $~ 300$ million people worldwide, causes greater overall disability in the UK than diabetes or breast cancer, ${ }^{1}$ and places a huge burden on healthcare resources. ${ }^{3}$ Cystic fibrosis (CF), the commonest genetic illness in the UK, is a life-limiting illness affecting children and young adults and is associated with high costs of daily care. ${ }^{4}$ These chronic airways diseases differ substantially in underlying pathophysiology, but are all characterized by airway inflammation, airflow obstruction, and high morbidity and mortality. ${ }^{1}$

In recent years, there has been significant progress towards understanding the biology underlying airways diseases. In asthma and COPD, the identification of distinct inflammatory phenotypes and biomarkers such as fractional exhaled nitric oxide (FeNO) and blood eosinophil counts has allowed the development of highly-targeted biological drugs. ${ }^{5-7}$ In CF, novel drugs targeting specific CFTR mutations have revolutionized the management of some patients. ${ }^{8,9}$ Such targeted therapy, the essence of precision medicine, is becoming increasingly feasible across the spectrum of airways diseases.

Unfortunately, the assessment of lung function in airways diseases has not kept pace with these exciting scientific and therapeutic advances. We remain heavily reliant on traditional spirometric measures such as forced expiratory volume in 1 second $\left(\mathrm{FEV}_{1}\right)$ and forced vital capacity (FVC) for diagnosis and disease monitoring, despite an increasing recognition that they fail to detect subtle disease, are insensitive to treatment effects and cannot capture the complexity of heterogeneous 
pathology. In this review, we outline the challenges this presents for the respiratory community, focusing particularly on: i) early diagnosis; ii) identification of specific phenotypes or 'treatable traits' in patients with established disease, and iii) identification of response to treatment. We provide an overview of established techniques for measuring lung function, a more detailed account of which is provided elsewhere ${ }^{10}$, before discussing emerging techniques that may help to overcome these clinical challenges.

\section{CURRENT CHALLENGES IN AIRWAYS DISEASES}

\section{COPD}

A major challenge to efforts to modify the natural history of COPD is identification of early disease. Guidelines advocate the demonstration of obstructive spirometry (post-bronchodilation $\left.\mathrm{FEV}_{1} / \mathrm{FVC}<0.70\right)^{11}$ for diagnosis, but $\mathrm{FEV}_{1}$ reflects predominantly large airway dysfunction, and may miss active disease in the smaller ( $<2 \mathrm{~mm}$ diameter) airways. In younger patients, this may lead either to failure of diagnosis despite significant symptoms or to late diagnosis when structural changes in the lung may be irreversible. In either case, important opportunities for early therapy (i.e. inhaled corticosteroids for eosinophilic disease) ${ }^{12}$ or secondary prevention (including smoking cessation) are missed, ${ }^{13,14}$ highlighting the need for markers of early disease. ${ }^{15}$ In older patients, the opposite may occur when $\mathrm{FEV}_{1} / \mathrm{FVC}<0.70$ is used as a diagnostic criterion: overdiagnosis and unnecessary treatment ${ }^{16}$. It has been proposed that classification should be based on a lower limit of normal (LLN) i.e., more than 1.64 SD below the predicted level $\left(5^{\text {th }}\right.$ percentile), which should add accuracy and confidence to the diagnostic approach, but this recommendation has not been taken up by global guidelines ${ }^{16,17}$.

A second challenge is stratification. $\mathrm{FEV}_{1}$ remains the principal means of classifying COPD severity, and it is is predictive of mortality and outcomes at a population level. ${ }^{18}$ However, its correlation with symptoms and key clinical outcomes such as exacerbation frequency and treatment 
response is poor, as recognized by GOLD in its recent updated statement. ${ }^{11}$ This may reflect the inability of spirometry to capture or adequately distinguish heterogeneous underlying pathologies, for example obstructive bronchitis, emphysema, or the progression of childhood/chronic asthma. Finally, it is now widely acknowledged that fixed airflow obstruction in later adult life can be the result of several different lung-function decline trajectories. ${ }^{19}$ Without the ability to discriminate between these trajectories, it will be difficult to identify true diseasemodifying interventions.

\section{Asthma}

An important but under-appreciated feature of asthma is the discordance between lung function measures and clinical markers of disease control, including symptoms, exacerbation frequency and response to treatment. Baseline $\mathrm{FEV}_{1}$, for example, correlates poorly with symptom severity and quality of life, ${ }^{20}$ and may also be dissociated from markers of airway inflammation such as FeNO, ${ }^{21}$ perhaps reflecting the poor sensitivity of spirometry for disease located in the small airways.

Asthma treatment guidelines rely on a step-wise increase or decrease in medications based on symptom control ${ }^{22}$ but patients with asthma vary in their underlying biology and response to treatment, so this approach often leads to poor targeting of treatment. Classification into inflammatory phenotypes based on 'type-2' biomarkers reflecting type-2 cytokine activity (blood/sputum eosinophils and FeNO) has been useful in understanding and targeting therapies, ${ }^{23-26}$ but does not always predict response. Some patients with type-2 high inflammation, for example, do not respond to inhaled corticosteroids and require systemic treatment for disease control. This may, in part, reflect a more peripheral distribution of disease, inaccessible to inhaled corticosteroids. ${ }^{27,28}$ Current measures of lung pathophysiology cannot identify this phenotype. 
The advent of targeted biological therapy offers new opportunities in severe asthma, with early evidence of improvement in quality of life, reduction in oral corticosteroid use and decreased frequency of exacerbations. . $^{5,629,30}$ However, biologics are expensive and further advances will require careful patient selection and evaluation of efficacy, including the withdrawal of ineffective therapy. Again, baseline indices often provide a limited perspective on long-term efficacy, leading some authors to suggest that the crude "global evaluation of treatment effectiveness" by physicians as the best measure of response. ${ }^{31}$ Clearly, more objective measurements of disease activity would be helpful.

\section{Cystic fibrosis}

Spirometry remains the principal means of monitoring CF progression, but advances in multidisciplinary care mean that many patients maintain a normal $\mathrm{FEV}_{1}$ well into adulthood. There is mounting evidence that bronchiectasis and airway obstruction may go undetected in earlier life, ${ }^{32}$ preventing early intervention. The advent of highly effective but expensive new CFTR modulators ${ }^{8,9}$ has highlighted the urgent need for more sensitive assays of lung function, not only for timely introduction of therapy, but also to monitor response, and consider withdrawal in those who do not respond.

There is also a particular need for earlier detection of deteriorating lung function in CF patients, which may precede clinical exacerbations. Such episodes are important events, from which patients may not fully recover, leading to progressive lung function decline. ${ }^{33}$ Prompt and effective treatment is paramount, and is currently limited by reliance on changes in FEV . $_{\text {. }}$

\section{PULMONARY FUNCTION TESTS USED ROUTINELY IN CLINICAL PRACTICE}


The utility of the routine pulmonary function tests in assessing airways diseases, together with

their advantages and disadvantages are detailed in Table 1.

Table 1: Advantages and disadvantages of current routinely-used pulmonary function tests

\begin{tabular}{|c|c|c|}
\hline Method and measures & Advantages & Disadvantages \\
\hline $\begin{array}{l}\text { Spirometry } \\
\text { FEV1 } \\
\text { FVC } \\
\text { FEV1/FVC } \\
\text { FEV1/VC }\end{array}$ & 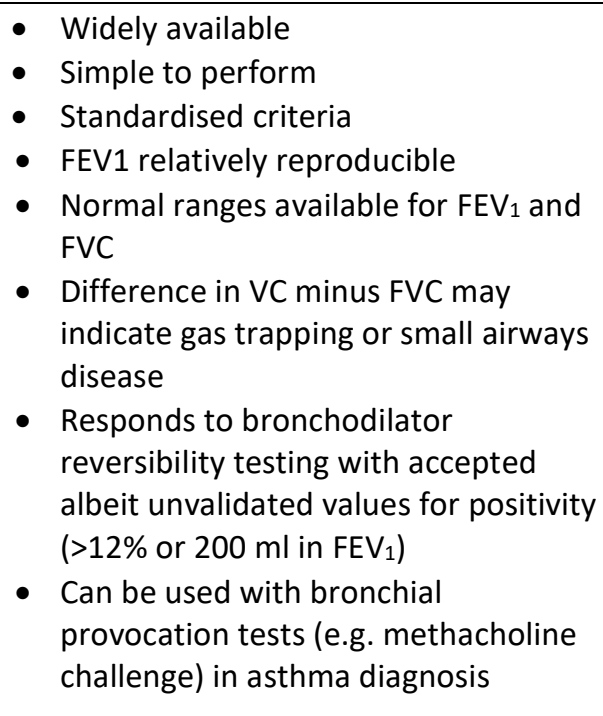 & $\begin{array}{l}\text { - } \begin{array}{l}\text { Partly effort- and technique- } \\
\text { dependent }\end{array} \\
\text { - Insensitivity of FEV } \mathrm{F}_{1} \text { to early disease } \\
\text { and small airways dysfunction } \\
\text { - } \\
\text { - } \text { Gatural decline in } \mathrm{FEV}_{1} \text { with age } \\
\text { apparently normal FEV1/FVC* } \\
\text { - Low sensitivity of bronchodilator } \\
\text { reversibility in asthma diagnosis }\end{array}$ \\
\hline $\begin{array}{l}\text { Flow-volume loop } \\
\text { MMEF (FEF } 25-75 \%)\end{array}$ & $\begin{array}{l}\text { - Easily obtainable from spirometry } \\
\text { - Sensitive to small airway obstruction } \\
\text { - May identify pre-COPD pathology } \\
\text { (e.g. GOLD stage } 0)^{34} \\
\text { - Can identify extra-thoracic } \\
\text { obstruction }\end{array}$ & $\begin{array}{l}\text { - Shape of loop useful but subjective } \\
\text { - } \text { and not quantifiable } \\
\text { - } \quad \text { Wide range of normal values, LLN } \\
\text { at }<60 \% \text { predicted }{ }^{35} \\
\text { - } \text { Dependence on FVC } \\
\text { - } \text { Poor reproducibility } \\
\text { - } \text { Low sensitivity if FEV } \mathrm{F}_{1} / \mathrm{FVC}>75 \%^{36} \\
\text { - } \text { Poor correlation with other } \\
\text { measures of small airway } \\
\text { dysfunction }{ }^{37} \\
\end{array}$ \\
\hline $\begin{array}{l}\text { Body plethysmography } \\
\text { TLC } \\
\text { RV } \\
\text { FRC } \\
\text { RV/TLC } \\
\text { Raw }\end{array}$ & $\begin{array}{l}\text { - } \quad \text { Identification of hyperinflation } \\
\text { - } \quad \mathrm{RV} \text { is sensitive to small airways } \\
\text { resistance and inflammation in } \\
\text { asthma }{ }^{38} \text { and COPD } \text { CO }^{39} \\
\text { - } \quad \mathrm{RV} / \mathrm{TLC} \text { is a marker of gas trapping in } \\
\text { severe disease }\end{array}$ & $\begin{array}{l}\text { - Effort dependent } \\
\text { - Relatively time-consuming } \\
\text { - May over-estimate lung volumes } \\
\text { when significant gas trapping is } \\
\text { present }^{40}\end{array}$ \\
\hline $\begin{array}{l}\text { Gas Transfer or } \\
\text { Diffusing Capacity } \\
\text { TLCO } \\
\text { KCO } \\
\text { VA }\end{array}$ & $\begin{array}{l}\text { - Helps identify degree of } \\
\text { emphysematous change in COPD, } \\
\text { with fall in TLCO or KCO correlating } \\
\text { with the extent of emphysema }{ }^{41} \\
\text { - Allows determination of lung function } \\
\text { decline when spirometry is normal }\end{array}$ & $\begin{array}{l}\text { - Difficulty of breath-holding for } \\
\text { breathless patients } \\
\text { - Temporal monitoring needs } \\
\text { adjustment for changes in } \\
\text { haemoglobin or blood CO } \\
\text { - Extra-thoracic factors e.g. muscle } \\
\text { weakness or obesity may increase } \\
\text { KCO by lowering VA } \\
\text { - Cannot be used in oxygen- } \\
\text { dependent patients }\end{array}$ \\
\hline
\end{tabular}

$\mathrm{FEV}_{1}$, forced expiratory volume of gas in 1 second; $\mathrm{FVC}$, the maximum volume of gas forcefully expired from total lung capacity (TLC) to residual volume (RV); VC, vital capacity the maximum volume of gas expired during a slow expiratory maneuver; MMEF 
or $\mathrm{FEF}_{25-75 \%}$, mean flow between maximal flow at $25 \%$ of FVC and $75 \%$ of FVC; LLN, lower limit of normal; FRC, functional residual capacity; Raw, airways resistance; TLCO, gas transfer factor signifying a measure of CO uptake by the whole lung; VA, accessible alveolar volume during gas transfer measurement; $\mathrm{KCO}$, measure of the rate of CO uptake from alveolar gas to blood, arithmetically equal to TLCO/VA. *While gas trapping may cause a falsely normal FEV 1 /FVC, FEV1/VC will still indicate obstruction in this scenario; however this means additional slow expiratory maneuvers need to be performed (in triplicate) which adds time and discomfort for patient.

Spirometry is the most routinely and widely-used lung function test in the diagnosis and monitoring of airways diseases (Figure 1a). The FEV1/FVC ratio is used to diagnose airflow obstruction, and the \%predicted FEV 1 to classify severity and monitor disease progression. Important information is also acquired from the flow-volume loop (Figure 1b): a concave expiratory loop shape and a low maximal mid-expiratory flow (MMEF) index indicate small airways dysfunction, even when $\mathrm{FEV}_{1}$ or $\mathrm{FEV}_{1} / \mathrm{FVC}$ are normal.

Body plethysmography measures static lung volumes and provides indices such as: Total Lung Capacity (TLC), Residual Volume (RV), Functional Residual Capacity (FRC), RV/TLC and Airways Resistance (Raw). It is useful in detecting hyperinflation, i.e. increased lung volume at endexpiration, which may be caused by airway narrowing in either asthma or COPD, or by loss of elastic recoil in emphysema, leading to early airway closure and gas trapping. In COPD, body plethysmography is routinely used to guide management of hyperinflation using lung volume reduction therapies such endobronchial valves or coils. ${ }^{42,43}$ In asthma, body plethysmography can inaccurately overestimate lung volumes ${ }^{40}$ although measures such as \%predicted RV and RV/TLC may correlate with small airways disease ${ }^{38}$ and gas trapping in severe disease ${ }^{37}$. Additionally, measurement of specific airways resistance (sRaw, ratio of shift volume-to flow rate) and airways resistance (Raw, alveolar pressure change-to flow rate, arithmetically equal to sRaw/FRC) can be obtained from resistance-volume loops. It has been suggested that these measurements may be useful in COPD patients with hyperinflation and that they are more sensitive to change following bronchodilation or bronchial provocation tests. ${ }^{44}$ However, these potential advantages need to be set against measurement complexity and increased within-subject variability. 
Gas transfer factor quantifies the transfer of carbon monoxide (CO) from the alveolar gas into the pulmonary capillaries. Measurement involves inhalation of a gas mixture containing a low concentration of $\mathrm{CO}$, plus a known concentration of an inert gas, e.g. helium. During a single 10second breath hold, the rate of CO uptake from alveolar gas into capillary blood (KCO) is measured, and the accessible lung volume (VA) is estimated by helium dilution. TLCO is calculated as KCO x VA, and essentially represents an index of whole lung CO transfer from gas to blood. Transfer factor is useful in distinguishing conditions characterized by alveolar destruction, e.g. emphysematous COPD, from those with preserved lung architecture, e.g. asthma. In asthma and CF, TLCO and KCO are typically preserved or may even be elevated, due to either increased pulmonary blood flow (which increases TLCO and KCO), or reduced apparent VA (which disproportionately increases in $\mathrm{KCO}$ ). Such $\mathrm{KCO}$ changes may be useful markers of early or heterogeneous disease ${ }^{45} \mathrm{~A}$ new, but similar, measure is the transfer factor for nitric oxide (TLNO), which is 4.5-5.0 times the TLCO value. Unlike TLCO, TLNO is unaffected by the partial pressure of alveolar oxygen or haemoglobin concentration ${ }^{46}$. In practice, TLNO and TLCO are measured simultaneously, and the TLNO/TLCO ratio may prove to have some diagnostic advantage over the TLCO alone ${ }^{47}$.

\section{PHYSIOLOGICAL ASSESSMENTS OF LUNG FUNCTION CURRENTLY NOT IN ROUTINE USE}

A number of physiological techniques, detailed below, have been explored as tools for the assessment of small airways function or the detection of early or subtle lung damage.

\section{Oscillometry techniques}

Forced oscillometry techniques (FOT), ${ }^{48-50}$ first described in $1956,{ }^{48}$ apply oscillating pressure airwaves of multiple frequencies $(3-30 \mathrm{~Hz})$ during normal tidal breathing. Impulse Oscillometry is a form of FOT in which a fixed $5 \mathrm{~Hz}$ square pressure wave is applied and from which other frequencies are derived. The resulting changes in pressure and flow at the mouth are analysed 
using Fourier transformation to calculate parameters including Resistance (Rrs) and Reactance (Xrs), the latter predominantly reflecting elasticity of the peripheral lung parenchyma and chest wall (which also affects Rrs to a smaller extent). Low frequencies (e.g. $5 \mathrm{~Hz}$ ) penetrate deep into the lung, while high frequencies (e.g. $20 \mathrm{~Hz}$ ) are absorbed before reaching the smaller peripheral airways. Hence, R5 represents the total airway resistance, R20 the central airways resistance and R5-R20 is an index of small airway resistance.

A predominant increase of Rrs measured by oscillometry at low frequencies is thought to reflect obstruction in distal airways in asthma and COPD. ${ }^{49,51-53}$ Reactance at low frequencies (e.g. $X_{5}$ ) is more negative in obstructive lung disease. Impulse oscillometry indices are sensitive to bronchodilator effects in COPD and asthma $a^{54-56}$ and inhaled corticosteroids in asthma. ${ }^{57}$ They may also be useful in monitoring recovery from exacerbation. ${ }^{58}$ In addition to whole-breath system analysis, breath-by-breath analysis using oscillometry provides additional measures of airflow limitation. For example, patients with COPD experience expiratory flow limitation (EFL) during tidal breathing, which is a major determinant of dynamic hyperinflation and dyspnoea. The difference between inspiratory and expiratory reactance ( $\Delta \mathrm{Xrs}$ ) measured by osillometry can reliably detect this EFL in COPD patients ${ }^{59}$. Additionally, inspiratory-expiratory $\Delta \mathrm{X}_{5}$ analysis differentiated patients with asthma from those with COPD, presumably reflecting enhanced dynamic airway narrowing on expiration in $\mathrm{COPD}^{60}$.

The technique is non-invasive, easy to perform, effort-independent and reproducible. It is particularly attractive in small children, but has some disadvantages. Interference from swallowing and upper airway artifacts is common and to avoid these coaching and repetition may be required. While oscillometry measurements are portrayed as a marker of small airway dysfunction, the relevant indices may also be influenced by large airway abnormalities. Furthermore, universal normal reference ranges have not been established. Moreover, the interpretation of measurements and results' meaning is not straightforward for users. These 
disadvantages may explain why, although the equipment is widely available commercially, it has not been widely adopted by clinical lung function laboratories.

\section{Inert gas washout tests}

It was recognized in the 1950s that Multiple Breath Inert Gas Washout (MBW) could provide an index of ventilatory inhomogeneity, ${ }^{61}$ and shown almost 40 years ago that this technique might be helpful in the identification of patients with early lung disease. ${ }^{62}$ However, only since the development of modern gas analysers and computers has this approach become feasible in clinical practice. MBW measures ventilation inhomogeneity by tracking the washout of an endogenous (e.g. nitrogen) or exogenous (e.g. $\mathrm{SF}_{6}$ ) inert gas, with the latter requiring an initial wash-in phase. ${ }^{63,64}$ In the case of nitrogen washout, a subject breathes $100 \%$ oxygen, and the composition of exhaled gas is measured using a respiratory mass spectrometer or other device. Since nitrogen will be washed out rapidly from well-ventilated areas and more slowly from poorlyventilated areas, the overall rate and shape of nitrogen washout is a function of ventilation inhomogeneity (Figure 2).

This technique has been most widely used in patients with $\mathrm{CF}$, for whom recent advances in treatment have strengthened the rationale for identifying early disease. ${ }^{65} \mathrm{In}$ this setting, the most commonly used inhomogeneity index is the Lung Clearance Index $(\mathrm{LCl})^{63,66}$. To measure the $\mathrm{LCl}$, the washout begins at FRC, and continues until the concentration of tracer gas (e.g. nitrogen) has fallen to $1 / 40$ th of its original concentration. $\mathrm{LCl}$ is defined as the number of lung turnovers or FRC equivalents required to produce this fall in tracer gas concentration (see Figure 2 for details). In adults or adolescents, a fixed tidal volume is typically used to minimize changes in the deadspaceto-tidal volume ratio, which may influence $\mathrm{LCl}$ independently of inhomogeneity. In younger children, this is usually not possible, and measurements are made during normal tidal breathing. 
The LCl has attracted particular attention in children with CF, for whom it is easier than forced spirometry. ${ }^{66,67} \mathrm{LCl}$ identified abnormal lung function more readily than either plethysmography or spirometry in children with CF ( $73 \%$ compared to $47 \%$ and $13 \%$, respectively), and was higher in children with Pseudomonas colonization. ${ }^{67}$ Similarly, LCl was more useful than spirometry for tracking CF progression over time in preschool patients, ${ }^{68}$ and was more sensitive than $\mathrm{FEV}_{1}$ in detecting response to Ivacaftor in patients with mild lung disease. ${ }^{69}$ In contrast, in a group 110 children with CF, LCl failed to detect significant effects of CFTR gene therapy, despite small rises in $\mathrm{FEV}_{1} \cdot{ }^{70}$ Of note, however, $\mathrm{LCl}$ did identify a treatment effect in a patient subgroup with mild disease at baseline, in whom $\mathrm{FEV}_{1}$ did not change. This reinforces the likely role for $\mathrm{LCl}$ in patients with preserved spirometry, rather than those with established disease.

The LCI provides a global measure of ventilation inhomogeneity, but other MBW-derived indices such as Scond and Sacin, have been used to classify inhomogeneity as being either convectiondependent (occurring in the conductive airways) or diffusion-convection-dependent (occurring in the acinar airways), respectively. These indices are derived from a normalised nitrogen phase III slope analysis for each exhalation during the $\mathrm{MBW}^{71,72}$ (Figure 2). In smokers, changes in Sacin and Scond predated changes in spirometry, ${ }^{73}$ and Scond showed sustained reversibility with smoking cessation. ${ }^{74}$ In COPD, Scond correlated with $\mathrm{FEV}_{1}$, whilst Sacin correlated with diffusing capacity. ${ }^{75}$ In asthma, inhomogeneity indices correlated with airway inflammation, severity and asthma control, ${ }^{76-78}$ and have shown promise in predicting hyper-responsiveness and response to inhaled corticosteroids. ${ }^{79-81}$

Despite these promising results, MBW and its derived indices have limitations. As reflected in a recent ERS/ATS Consensus Statement, ${ }^{63}$ work is needed to improve the standardization and specification of equipment, procedures, and software/analytical algorithms..$^{82,83}$ In particular, some equipment is associated with considerable technical noise. ${ }^{84}$ Other limitations include the 
dependence of the $\mathrm{LCl}$ on just two points from the washout for its derivation, the requirement for washouts to be performed in triplicate, and the lack of consensus regarding standard ranges ${ }^{63,82}$. Finally, the $\mathrm{LCl}$ cannot differentiate between structural damage such as bronchiectasis, and airway narrowing due to mucus secretion. To date, these complexities have largely limited MBW to the research setting, other than in a few paediatric CF centres.

\section{Multiple Inert Gas Elimination Technique}

The Multiple Inert Gas Elimination Technique (MIGET) was developed in the 1970s and measures the pulmonary exchange of six different inert gases. ${ }^{85}$ These gases are simultaneously intravenously infused and when in steady state within the lung, their concentrations are measured in mixed expired gas and arterial blood, using gas chromatography. The measurements are used with a mathematical model of the lung to compute ventilation-perfusion distributions that best explain the simultaneous exchange of these gases. As such, MIGET can quantify ventilation-perfusion $(\mathrm{V} / \mathrm{Q})$ inequalities and pulmonary shunts.

This technique has significantly advanced our understanding of pulmonary pathophysiology. ${ }^{86}$ In patients with COPD, MIGET identified three distinct patterns of V/Q distribution (Figure 3). ${ }^{87,88}$ Patients with the so-called 'pink puffer' phenotype, for example, generally demonstrated large amounts of ventilation to high $V / Q$ areas, with minimal ventilation of very low $V / Q$ regions, presumably reflecting predominant emphysema and alveolar wall destruction. The correlation between V/Q distribution and clinical picture was less striking for other phenotypes, but MIGET clearly has the potential to identify important sub-populations. ${ }^{87}$ It also appears to be a sensitive marker of early disease in COPD, with substantial V/Q abnormalities reported in patients with only mild spirometric disease (GOLD stage 1 ) ${ }^{89}$ In asthma, asymptomatic patients with mild airway obstruction similarly had extensive V/Q mismatch using MIGET, with a distinct mode of low $\mathrm{V} / \mathrm{Q}$ units. ${ }^{90}$ Furthermore, during acute exacerbations $\mathrm{V} / \mathrm{Q}$ inequality was unrelated to 
spirometric abnormality, suggesting that factors other than bronchoconstriction, such as small airway mucous plugging, make an important contribution to V/Q imbalance. ${ }^{91,92}$

Despite these important advantages over conventional tests, MIGET is rarely used in the clinical setting, primarily due to its complexity and invasive nature. It is currently available only in a few expert research centres.

\section{EMERGING TECHNIQUES FOR ASSESSING LUNG PATHOPHYSIOLOGY}

\section{Lung imaging}

Quantitative computed tomography (CT)

CT imaging has made an important contribution to the diagnosis and management of airways disease for many years. It allows the identification of emphysema and bronchiectasis in COPD and $\mathrm{CF}$, respectively, and demonstrates significant expiratory gas trapping in asthma ${ }^{93,94}$. Its advantages include widespread availability, excellent spatial and temporal resolution, and rapid image acquisition. Important limitations include the lack of immediate accessibility in the clinic environment, the relatively subjective nature of reporting, and the need for ionizing radiation. On the latter point, low- and ultralow-dose CT scanning is emerging as a robust alternative to traditional protocols, and may permit more regular and widespread use of CT in the future. ${ }^{95}$

In relation to subjectivity, so-called 'quantitative $C T$ ' $(\mathrm{QCT})$ is beginning to address this issue. This technique uses commercial or bespoke software to quantify, either at the lobar or the whole lung level, the extent of emphysema, based on voxel-by-voxel attenuation; the degree of bronchial wall thickening, based on the ratio of average lumen diameter to wall area in the small airways; and the extent of gas trapping, based on differences between inspiratory and expiratory phase images. ${ }^{96-101}$ 
The extent of the correlation between QCT indices and conventional markers of disease severity appears variable, ${ }^{96,97}$ but importantly, QCT indices correlate independently with clinical outcomes including disease progression, mortality and symptoms. ${ }^{98,99}$ Furthermore, some radiological phenotypes appear to correspond well with particular genetic risk factors, raising the possibility that early CT might guide prognosis and management. ${ }^{100}$ Finally, there may be scope for combining CT with functional ${ }^{18} \mathrm{~F}-\mathrm{FDG}$ PET imaging, which has shown some promise in the identification of local inflammation in patients with asthma, ${ }^{102}$ raising the prospect of 'functional' CT imaging of airways pathophysiology.

\section{Magnetic resonance imaging (MRI)}

In contrast to $\mathrm{CT}$, thoracic MRI has historically played a minor part in respiratory medicine, due to the lack of parenchymal resolution. ${ }^{103}$ However, functional MRI using inhaled hyperpolarized noble gases such as helium-3 $\left({ }^{3} \mathrm{He}\right)$ and xenon-129 $\left({ }^{129} \mathrm{Xe}\right)$ has recently gained momentum in the research setting. Based on the intensity of gas signal in each voxel during a relatively brief breath hold, the distribution of hyperpolarised gas can be quantified as the 'percentage ventilated volume', highlighting ventilation defects due to bronchoconstriction or airway inflammation, or the 'apparent diffusion coefficient' (ADC), which serves as an index of the extent of parenchymal destruction, for example due to emphysema (Figure 4$)^{104}$.

In patients with asthma, hyperpolarized gas MRI indices of ventilation heterogeneity were increased compared with healthy volunteers, and responded to treatment with bronchodilation or bronchial thermoplasty. ${ }^{105}$ In a recent study, while heterogeneity assessed by ${ }^{3} \mathrm{He} \mathrm{MRI}$ improved significantly after bronchodilation in patients with severe asthma, residual heterogeneity was greater in those with poorly-controlled eosinophilic disease. ${ }^{106}$ In CF, heterogeneity can be observed prior to changes in $\mathrm{FEV}_{1}{ }^{107}$ while in COPD, there is variable 
correlation between hyperpolarised MRI imaging parameters and conventional lung function measures. ${ }^{96,108}$

Overall, hyperpolarized gas MRI holds considerable promise as a means of non-invasively estimating lung heterogeneity, perhaps even allowing assessment of gas transfer ${ }^{103}$ and providing phenotypic information beyond simple correlation with existing parameters. However, important caveats include the limited availability in the clinical setting and high cost. These limitations may be overcome in the future through the use of more widely-available contrast agents e.g. oxygen, in combination with standard proton MRI. A recent study in healthy volunteers reported good correlation between $\mathrm{V} / \mathrm{Q}$ relationships derived by from single slice proton MRI and those derived from MIGET. ${ }^{109}$

\section{Laser gas absorption spectroscopy and mathematical modeling of lung inhomogeneity}

Evidence from MIGET, MBW and imaging studies supports the notion that measuring gasexchange inhomogeneity within the lung may be useful in the diagnosis and management of airways diseases, as inhomogeneity measures are likely to be more sensitive to disease change. Nevertheless, these methodologies have yet to make a significant impact in the clinical setting.

Recently, a novel technology for highly precise in-airway gas analysis has been developed. ${ }^{110}$ This device accurately measures respiratory flows, and uses laser absorption spectroscopy to assess the composition of respired gases with substantially greater precision than any other available device. ${ }^{110}$ This level of precision has facilitated the development of a novel mathematical model of gas exchange within the lung, which uses data from a nitrogen MBW (10 min air, 5 min oxygen) to recover parameters reflecting multiple aspects of gas-exchange inhomogeneity. ${ }^{111}$ Briefly, the model divides the lung into many (125) subunits, each with an equal fractional share of total alveolar volume $\left(V_{A}\right)$ at $F R C$, but a different fractional share of total lung compliance $\left(C_{L}\right)$, 
deadspace $\left(V_{D}\right)$ and pulmonary vascular conductance $\left(C_{d}\right)$, and returns distributions for each of these properties, relative to volume, across the lung. The standard deviations of these distributions, $\sigma V_{D}: V_{A}$ for the deadspace distribution, and $\sigma C_{\mathrm{L}}: V_{A}$ and $\sigma C_{d}: V_{A}$ for compliance and conductance, respectively, quantify the degree of inhomogeneity. Total alveolar volume $\left(\mathrm{V}_{\text {Atot }}\right)$, total anatomical deadspace $\left(\mathrm{V}_{\mathrm{Dtot}}\right)$ and shunt fraction are also calculated, and $\mathrm{V} / \mathrm{Q}$ distributions similar to those derived from MIGET can be derived. ${ }^{111}$

This technique has important advantages over existing methods of measuring inhomogeneity. Unlike MIGET, it is non-invasive and simple to perform, both for the operator and for the patient and is appropriate for the outpatient clinic or lung function laboratory setting. In contrast to imaging methodologies, it does not use ionizing radiation, or require expensive scanners and reagents. In comparison with standard MBW techniques, it does not depend on the pattern of breathing and has significantly superior precision. ${ }^{110}$ For example, the typical bias in flow sensing between inspiration and expiration is an order of magnitude less (from $\sim 5 \%$ to $<0.2 \%$ ) than with standard MBW equipment. It is this high precision that has made the novel approach to modeling inhomogeneity possible. Furthermore, the inhomogeneity indices obtained are computed using the whole gas-exchange dataset (with datapoints every $10 \mathrm{~ms}$ ), unlike conventional MBW-derived indices (e.g. $\mathrm{LCl}$, Scond and Sacin), which rely on parameterization of the nitrogen washout profiles at specific time-points. From a physiological perspective, the unique partitioning of inhomogeneity into variations in deadspace and compliance, which are intrinsic structural properties of the lung, has the potential to provide important biological insights. It may, for example, be particularly well-placed to distinguish irreversible bronchiectasis (which may affect deadspace volume and variance) from reversible factors such as mucus plugging and airway inflammation (which may affect alveolar volume and the variance in lung compliance), or alternatively provide a novel sensitive marker of progressive emphysema, as suggested in preliminary studies. ${ }^{111}$ 
To date, it has been demonstrated that this technique can discriminate between healthy young volunteers, healthy elderly volunteers and patients with GOLD Stage 1-2 COPD (Figure 5), with excellent repeatability. ${ }^{111}$ Preliminary results suggests that certain lung inhomogeneity markers may also be sensitive markers of disease in other conditions: compared with healthy controls, $\sigma \mathrm{C}_{\mathrm{L}}: \mathrm{V}_{\mathrm{A}}$ is abnormally high in patients with asthma but preserved spirometry, ${ }^{112}$ and is also elevated in patients with CF and young healthy smokers with normal spirometry (Talbot NP et al, 2018: authors' preliminary data). The latter observation suggests that $\sigma C_{L}: V_{A}$ may provide a useful marker for assessing small airway dysfunction or early disease in the "unobstructed smoker".

These data suggest that this novel technique holds significant promise for the early detection of lung damage, disease stratification and monitoring of disease progression or regression. Larger studies are required to determine normative ranges in health and disease, and to assess the potential to address specific clinically relevant questions. As the technique is in its infancy, particular shortcomings are not yet clear; at present limitations include a relatively long measurement time (15 minutes), a requirement for excellent nose and mouth seal throughout the test, and an apparatus deadspace that is currently too large for use in young children. These limitations are likely to be surmountable with further engineering.

\section{CONCLUSIONS: TOWARDS PRECISION MEDICINE}

To improve outcomes in airways diseases and accelerate research and development of new disease-modifying drugs that can target "treatable traits", we must improve our ability to phenotype/endotype patients and detect disease activity earlier so that we can evaluate interventions that potentially modify its trajectory, and track disease activity (progression or regression) with greater sensitivity. This requires (a) improvements in physiological measurements of disease activity in the lung, such that tests are both sensitive to small changes 
and able to find patterns of abnormalities specific for particular airway or parenchymal pathology; and (b) a recognition that different test modalities, e.g. lung function, inflammatory/molecular phenotyping and imaging offer different insights into disease process. Appropriate Integration of these test modalities will improve our ability to phenotype patients and allow us to provide the 'right treatment to the right patient at the right time'.

\section{FUNDING}

This work was supported by the NIHR Biomedical Research Centre, Oxford.

\section{CONTRIBUTORSHIP}

NP and NPT contributed equally to this manuscript. All authors were responsible for the conception and design of the work. NP and NPT drafted the manuscript. All authors contributed significantly to revising the work and approved the final version published.

\section{COMPETING INTERESTS}

There are no competing interests in relation to this review to declare.

\section{EXCLUSIVE LICENSE}

This article is made available under the Creative Commons Attribution (CC BY) license.

\section{FIGURE LEGENDS}

Figure 1: (a) Volume-time graph obtained during a spirometry test, with volume of gas exhaled during a forced expiration against time, for a healthy volunteer (blue) and a patient with airway obstruction (red). FEV $\mathrm{V}_{1}$ is the volume of gas that the subject is able to exhale in the first second of forced expiration; FVC is the total volume of gas the subject can exhale in one forced exhaled breath, from total lung capacity (TLC) to residual volume (RV). (b) Flow-volume loop obtained 
during a spirometry test in a healthy volunteer (blue). The expiration loop displays flow against volume during forced expiration from TLC to RV and the inspiration loop flow against volume during inspiration form RV to TLC. MMEF (or $\mathrm{FEF}_{25-75}$ ) signifies the mean expiratory flow between the flow at $25 \%$ of $\mathrm{FVC}\left(\mathrm{MEF}_{25}\right)$ and the flow at $75 \%$ of $\mathrm{FVC}\left(\mathrm{MEF}_{75}\right)$. The red trace indicates "scooping" of the expiratory loop, as seen in airway obstruction.

Figure 2: Nitrogen concentration and volume tracings as a function of time during a nitrogen multiple breath washout (MBW) test. During the washout, the subject breathes $100 \%$ oxygen, which causes a progressive reduction in nitrogen concentration in the lung as it is being replaced by oxygen. The rate and shape of nitrogen washout is a function of ventilation inhomogeneity. The Lung clearance index $(\mathrm{LCl})$ is the number of lung turnovers or FRC equivalents required to produce a fall in nitrogen concentration to $1 / 40^{\text {th }}$, and is calculated using: (1) $L C l=C E V / F R C$, where CEV signifies the cumulative expired volume; and (2) FRC $=V_{\text {tracer gas }} /\left(C_{\text {initial }}-C_{\text {end }}\right)$, where $V_{\text {tracer gas }}$ signifies the total volume of expired tracer gas and $\mathrm{C}_{\text {initial }}$ and $\mathrm{C}_{\text {end }}$ are the starting and final concentrations of tracer gas, respectively. This MBW was done during a provocation phase with histamine. The inset illustrates the alveolar slope phase III in Nitrogen concentration versus expired volume for breaths 1 and 20 (scaled to mean nitrogen expired concentration), which is seen to increase from breath 1 to 20 . The normalized phase III slopes are used in further analyses to compute Sacin and Scond. Figure reproduced from Verbanck S et al, 1997.

Figure 3: Ventilation-perfusion ratio distributions obtained using the Multiple Inert Elimination Technique (MIGET) for a healthy individual in (a) and two patients with COPD in (b) and (c). In (a), the distributions for both ventilation (open circles) and perfusion (closed circles) are narrow, unimodal and centered around a V/Q ratio of $1 . \ln (\mathrm{b})$, the ventilation distribution is bimodal with areas of high $\mathrm{V} / \mathrm{Q}$ ratio. This pattern is predominantly seen in patients with the emphysematoustype of COPD, likely due to alveolar wall destruction. In (c), the perfusion distribution is bimodally 
shaped with areas of low V/Q. This pattern is predominantly seen in patients with bronchitis-type of COPD, likely due to mucus plugging in airways. There is a third mixed pattern (not shown here), which likely represents the presence of both types of pathologies. Figure reproduced with permission from West JB, 2011.

Figure 4: Images obtained using thoracic magnetic resonance imaging, enhanced with hyperpolarized $\mathrm{Xe}^{129}$ (left) or $\mathrm{He}^{3}$ (right), in two healthy volunteers and two patients with COPD. The figures show the distribution of the 'apparent diffusion coefficient' (ADC), a measure of parenchymal destruction. The images reveal low values and a uniform distribution for ADC in healthy volunteers, but significant increased values with a markedly heterogenous distribution in patients with COPD. Hyperpolarised gas MRI imaging also allows visualization and quantitation of the distribution of static ventilation in patients without emphysema, in whom ventilation deficits reflect airway narrowing due to inflammation or bronchoconstriction (not shown here). Figure reproduced with permission from Mugler JP $3^{\text {rd }}$ et al, 2013.

Figure 5: Example distributions recovered by fitting the lung inhomogeneity model to the gasexchange data from a nitrogen MBW using the laser gas absorption spectroscopy device, for a healthy volunteer ( $A, C, E$ and $G$ ) and for a patient with GOLD Stage II COPD (B, D, F, and H). Shown are distributions for: ventilation:perfusion ratios $\left(\dot{V}_{A} / \dot{Q}\right)$ in $A \& B$; fractional compliance:volume ratios $\left(C_{\llcorner}: V_{A}\right)$ in $C \& D$; fractional vascular conductance:volume ratios $\left(C_{d}: V_{A}\right)$ in $E$ \& $F$ (lognormal); and scaled fractional deadspace:volume ratios $\left(V_{D}: V_{A}\right)$ in $G \& H$ (normal). Note that all distributions have larger variances in COPD, and estimates for shunt and deadspace are significantly higher in the COPD patient. Figure reproduced with data from Mountain JP et al, 2018.

\section{REFERENCES}


1. (WHO) WHO. Global surveillance, prevention and control of chronic respiratory diseases: a comprehensive approach; 2007.

2. Andersson F, Borg S, Jansson SA, et al. The costs of exacerbations in chronic obstructive pulmonary disease (COPD). Respir Med 2002;96(9):700-708.

3. O'Neill S, Sweeney J, Patterson CC, et al. The cost of treating severe refractory asthma in the UK: an economic analysis from the British Thoracic Society Difficult Asthma Registry. Thorax 2015;70(4):376-378.

4. Angelis A, Kanavos P, Lopez-Bastida J, et al. Social and economic costs and health-related quality of life in non-institutionalised patients with cystic fibrosis in the United Kingdom. BMC Health Serv Res 2015;15:428.

5. Castro M, Corren J, Pavord ID, et al. Dupilumab Efficacy and Safety in Moderate-to-Severe Uncontrolled Asthma. N Engl J Med 2018;378(26):2486-2496.

6. Haldar P, Brightling CE, Hargadon B, et al. Mepolizumab and exacerbations of refractory eosinophilic asthma. N Engl J Med 2009;360(10):973-984.

7. Pavord ID, Chanez P, Criner GJ, et al. Mepolizumab for Eosinophilic Chronic Obstructive Pulmonary Disease. N Engl J Med 2017;377(17):1613-1629.

8. Ramsey BW, Davies J, McElvaney NG, et al. A CFTR potentiator in patients with cystic fibrosis and the G551D mutation. N Engl J Med 2011;365(18):1663-1672.

9. Wainwright CE, Elborn JS, Ramsey BW, et al. Lumacaftor-Ivacaftor in Patients with Cystic Fibrosis Homozygous for Phe508del CFTR. N Engl J Med 2015;373(3):220231.

10. Hughes JMB. Physiology and Practice of Pulmonary Function: Association for Respiratory Technology \& Physiology; 2011.

11. (GOLD) GifC. Global Strategy for the diagnosis, management, and prevention of Chronic Obstructive Pulmonary Disease. 2018 Report 2018.

12. Hancox RJ, Pavord ID, Sears MR. Associations between blood eosinophils and decline in lung function among adults with and without asthma. Eur Respir $J$ 2018;51(4).

13. Mannino DM, Make BJ. Is it time to move beyond the "O" in early COPD? Eur Respir J 2015;46(6):1535-1537.

14. Woodruff PG, Barr RG, Bleecker E, et al. Clinical Significance of Symptoms in Smokers with Preserved Pulmonary Function. N Engl J Med 2016;374(19):1811-1821. 15. Martinez FJ, Han MK, Allinson JP, et al. At the Root: Defining and Halting Progression of Early Chronic Obstructive Pulmonary Disease. Am J Respir Crit Care Med 2018;197(12):1540-1551.

16. Quanjer PH, Ruppel G, Brusasco V, et al. COPD (confusion over proper diagnosis) in the zone of maximum uncertainty. Eur Respir J 2015;46(5):1523-1524.

17. Quanjer PH, Steenbruggen I, van den Berg JW. Diagnosis of airways obstruction should be based on symptoms and an FEV1/FVC ratio below the lower limit of normal. BMJ 2016;352:i397.

18. Anthonisen NR, Wright EC, Hodgkin JE. Prognosis in chronic obstructive pulmonary disease. Am Rev Respir Dis 1986;133(1):14-20.

19. Lange P, Celli B, Agusti A, et al. Lung-Function Trajectories Leading to Chronic Obstructive Pulmonary Disease. N Engl J Med 2015;373(2):111-122.

20. Scichilone N, Marchese R, Soresi S, et al. Deep inspiration-induced changes in lung volume decrease with severity of asthma. Respir Med 2007;101(5):951-956.

21. Lehtimaki L, Kankaanranta H, Saarelainen S, et al. Peripheral inflammation in patients with asthmatic symptoms but normal lung function. $J$ Asthma 2005;42(7):605-609. 
22. (GINA) GIfA. 2018 GINA Report, Global Strategy for Asthma Management and Prevention; 2018.

23. Haldar P, Pavord ID, Shaw DE, et al. Cluster analysis and clinical asthma phenotypes. Am J Respir Crit Care Med 2008;178(3):218-224.

24. Green RH, Brightling CE, McKenna S, et al. Asthma exacerbations and sputum eosinophil counts: a randomised controlled trial. Lancet 2002;360(9347):1715-1721.

25. Syk J, Malinovschi A, Johansson G, et al. Anti-inflammatory treatment of atopic asthma guided by exhaled nitric oxide: a randomized, controlled trial. J Allergy Clin Immunol Pract 2013;1(6):639-648 e631-638.

26. McNicholl DM, Stevenson M, McGarvey LP, et al. The utility of fractional exhaled nitric oxide suppression in the identification of nonadherence in difficult asthma. Am J Respir Crit Care Med 2012;186(11):1102-1108.

27. Berry M, Hargadon B, Morgan A, et al. Alveolar nitric oxide in adults with asthma: evidence of distal lung inflammation in refractory asthma. Eur Respir J 2005;25(6):986-991.

28. Hodgson D, Anderson J, Reynolds C, et al. A randomised controlled trial of small particle inhaled steroids in refractory eosinophilic asthma (SPIRA). Thorax 2015;70(6):559-565.

29. Busse W, Corren J, Lanier BQ, et al. Omalizumab, anti-IgE recombinant humanized monoclonal antibody, for the treatment of severe allergic asthma. J Allergy Clin Immunol 2001;108(2):184-190.

30. Rabe KF, Nair P, Brusselle G, et al. Efficacy and Safety of Dupilumab in Glucocorticoid-Dependent Severe Asthma. N Engl J Med 2018;378(26):2475-2485.

31. Bousquet J, Brusselle G, Buhl R, et al. Care pathways for the selection of a biologic in severe asthma. Eur Respir J 2017;50(6).

32. Wielputz MO, Puderbach M, Kopp-Schneider A, et al. Magnetic resonance imaging detects changes in structure and perfusion, and response to therapy in early cystic fibrosis lung disease. Am J Respir Crit Care Med 2014;189(8):956-965.

33. Sanders DB, Bittner RC, Rosenfeld M, et al. Failure to recover to baseline pulmonary function after cystic fibrosis pulmonary exacerbation. Am J Respir Crit Care Med 2010;182(5):627-632.

34. Mirsadraee M, Boskabady MH, Attaran D. Diagnosis of chronic obstructive pulmonary disease earlier than current Global Initiative for Obstructive Lung Disease guidelines using a feasible spirometry parameter (maximal-mid expiratory flow/forced vital capacity). Chron Respir Dis 2013;10(4):191-196.

35. Burgel PR. The role of small airways in obstructive airway diseases. Eur Respir Rev 2011;20(119):23-33.

36. Gelb AF, Williams AJ, Zamel N. Spirometry. FEV1 vs FEF25-75 percent. Chest 1983;84(4):473-474.

37. Sorkness RL, Bleecker ER, Busse WW, et al. Lung function in adults with stable but severe asthma: air trapping and incomplete reversal of obstruction with bronchodilation. J Appl Physiol (1985) 2008;104(2):394-403.

38. Kraft M, Pak J, Martin RJ, et al. Distal lung dysfunction at night in nocturnal asthma. Am J Respir Crit Care Med 2001;163(7):1551-1556.

39. Turato G, Zuin R, Miniati M, et al. Airway inflammation in severe chronic obstructive pulmonary disease: relationship with lung function and radiologic emphysema. Am J Respir Crit Care Med 2002;166(1):105-110. 
40. Stanescu DC, Rodenstein D, Cauberghs M, et al. Failure of body plethysmography in bronchial asthma. J Appl Physiol Respir Environ Exerc Physiol 1982;52(4):939-948.

41. Barjaktarevic I, Springmeyer S, Gonzalez X, et al. Diffusing capacity for carbon monoxide correlates best with tissue volume from quantitative CT scanning analysis. Chest 2015;147(6):1485-1493.

42. Sciurba FC, Criner GJ, Strange C, et al. Effect of Endobronchial Coils vs Usual Care on Exercise Tolerance in Patients With Severe Emphysema: The RENEW Randomized Clinical Trial. JAMA 2016;315(20):2178-2189.

43. Sciurba FC, Ernst A, Herth FJ, et al. A randomized study of endobronchial valves for advanced emphysema. N Engl J Med 2010;363(13):1233-1244.

44. Criee CP, Sorichter S, Smith HJ, et al. Body plethysmography--its principles and clinical use. Respir Med 2011;105(7):959-971.

45. Hughes JM, Pride NB. Examination of the carbon monoxide diffusing capacity (DL(CO)) in relation to its KCO and VA components. Am J Respir Crit Care Med 2012;186(2):132-139.

46. Zavorsky GS, van der Lee I. Can the measurement of pulmonary diffusing capacity for nitric oxide replace the measurement of pulmonary diffusing capacity for carbon monoxide? Respir Physiol Neurobiol 2017;241:9-16.

47. Hughes JMB, Dinh-Xuan AT. The DLNO/DLCO ratio: Physiological significance and clinical implications. Respir Physiol Neurobiol 2017;241:17-22.

48. Dubois AB, Brody AW, Lewis DH, et al. Oscillation mechanics of lungs and chest in man. J Appl Physiol 1956;8(6):587-594.

49. Goldman MD, Saadeh C, Ross D. Clinical applications of forced oscillation to assess peripheral airway function. Respir Physiol Neurobiol 2005;148(1-2):179-194.

50. Oostveen E, MacLeod D, Lorino H, et al. The forced oscillation technique in clinical practice: methodology, recommendations and future developments. Eur Respir J 2003;22(6):1026-1041.

51. Grimby G, Takishima T, Graham W, et al. Frequency dependence of flow resistance in patients with obstructive lung disease. J Clin Invest 1968;47(6):14551465.

52. Kolsum U, Borrill Z, Roy K, et al. Impulse oscillometry in COPD: identification of measurements related to airway obstruction, airway conductance and lung volumes. Respir Med 2009;103(1):136-143.

53. Cavalcanti JV, Lopes AJ, Jansen JM, et al. Detection of changes in respiratory mechanics due to increasing degrees of airway obstruction in asthma by the forced oscillation technique. Respir Med 2006;100(12):2207-2219.

54. Borrill ZL, Houghton CM, Woodcock AA, et al. Measuring bronchodilation in COPD clinical trials. Br J Clin Pharmacol 2005;59(4):379-384.

55. Houghton CM, Woodcock AA, Singh D. A comparison of lung function methods for assessing dose-response effects of salbutamol. $\mathrm{Br} J$ Clin Pharmacol 2004;58(2):134-141.

56. Park JW, Lee YW, Jung YH, et al. Impulse oscillometry for estimation of airway obstruction and bronchodilation in adults with mild obstructive asthma. Ann Allergy Asthma Immunol 2007;98(6):546-552.

57. Hoshino M. Comparison of effectiveness in ciclesonide and fluticasone propionate on small airway function in mild asthma. Allergol Int 2010;59(1):59-66. 
58. Johnson MK, Birch M, Carter R, et al. Measurement of physiological recovery from exacerbation of chronic obstructive pulmonary disease using within-breath forced oscillometry. Thorax 2007;62(4):299-306.

59. Dellaca RL, Santus P, Aliverti A, et al. Detection of expiratory flow limitation in COPD using the forced oscillation technique. Eur Respir J 2004;23(2):232-240.

60. Paredi P, Goldman M, Alamen A, et al. Comparison of inspiratory and expiratory resistance and reactance in patients with asthma and chronic obstructive pulmonary disease. Thorax 2010;65(3):263-267.

61. Fowler WS, Cornish ER, Jr., Kety SS. Lung function studies. VIII. Analysis of alveolar ventilation by pulmonary N2 clearance curves. J Clin Invest 1952;31(1):4050.

62. Fleming GM, Chester EH, Saniie J, et al. Ventilation inhomogeneity using multibreath nitrogen washout: comparison of moment ratios and other indexes. $\mathrm{Am}$ Rev Respir Dis 1980;121(5):789-794.

63. Robinson PD, Latzin P, Verbanck S, et al. Consensus statement for inert gas washout measurement using multiple- and single- breath tests. Eur Respir J 2013;41(3):507-522.

64. Robinson PD, Goldman MD, Gustafsson PM. Inert gas washout: theoretical background and clinical utility in respiratory disease. Respiration 2009;78(3):339355.

65. Elborn JS. Cystic fibrosis. Lancet 2016;388(10059):2519-2531.

66. Horsley AR, Gustafsson PM, Macleod KA, et al. Lung clearance index is a sensitive, repeatable and practical measure of airways disease in adults with cystic fibrosis. Thorax 2008;63(2):135-140.

67. Aurora P, Bush A, Gustafsson P, et al. Multiple-breath washout as a marker of lung disease in preschool children with cystic fibrosis. Am J Respir Crit Care Med 2005;171(3):249-256.

68. Stanojevic S, Davis SD, Retsch-Bogart G, et al. Progression of Lung Disease in Preschool Patients with Cystic Fibrosis. Am J Respir Crit Care Med 2017;195(9):12161225.

69. Davies J, Sheridan H, Bell N, et al. Assessment of clinical response to ivacaftor with lung clearance index in cystic fibrosis patients with a G551D-CFTR mutation and preserved spirometry: a randomised controlled trial. Lancet Respir Med 2013;1(8):630-638.

70. Alton E, Armstrong DK, Ashby D, et al. A randomised, double-blind, placebocontrolled trial of repeated nebulisation of non-viral cystic fibrosis transmembrane conductance regulator (CFTR) gene therapy in patients with cystic fibrosis. Southampton (UK); 2016.

71. Verbanck S, Paiva M. Gas mixing in the airways and airspaces. Compr Physiol 2011;1(2):809-834.

72. Verbanck S, Schuermans D, Van Muylem A, et al. Ventilation distribution during histamine provocation. J Appl Physiol (1985) 1997;83(6):1907-1916.

73. Verbanck S, Schuermans D, Meysman M, et al. Noninvasive assessment of airway alterations in smokers: the small airways revisited. Am J Respir Crit Care Med 2004;170(4):414-419.

74. Verbanck S, Schuermans D, Paiva M, et al. Small airway function improvement after smoking cessation in smokers without airway obstruction. Am J Respir Crit Care Med 2006;174(8):853-857. 
75. Verbanck S, Schuermans D, Van Muylem A, et al. Conductive and acinar lungzone contributions to ventilation inhomogeneity in COPD. Am J Respir Crit Care Med 1998;157(5 Pt 1):1573-1577.

76. Verbanck S, Schuermans D, Vincken W. Inflammation and airway function in the lung periphery of patients with stable asthma. J Allergy Clin Immunol 2010;125(3):611-616.

77. Thompson BR, Douglass JA, Ellis MJ, et al. Peripheral lung function in patients with stable and unstable asthma. J Allergy Clin Immunol 2013;131(5):1322-1328.

78. Macleod KA, Horsley AR, Bell NJ, et al. Ventilation heterogeneity in children with well controlled asthma with normal spirometry indicates residual airways disease. Thorax 2009;64(1):33-37.

79. Verbanck S, Schuermans D, Paiva M, et al. The functional benefit of antiinflammatory aerosols in the lung periphery. J Allergy Clin Immunol 2006;118(2):340346.

80. Scichilone N, Battaglia S, Sorino C, et al. Effects of extra-fine inhaled beclomethasone/formoterol on both large and small airways in asthma. Allergy 2010;65(7):897-902.

81. Farah CS, King GG, Brown NJ, et al. Ventilation heterogeneity predicts asthma control in adults following inhaled corticosteroid dose titration. J Allergy Clin Immunol 2012;130(1):61-68.

82. Summermatter S, Singer F, Latzin P, et al. Impact of Software Settings on Multiple-Breath Washout Outcomes. PLoS One 2015;10(7):e0132250.

83. Anagnostopoulou P, Yammine S, Schmidt A, et al. False normal Lung Clearance Index in infants with cystic fibrosis due to software algorithms. Pediatr Pulmonol 2015;50(10):970-977.

84. Tonga KO, Robinson PD, Farah CS, et al. In vitro and in vivo functional residual capacity comparisons between multiple-breath nitrogen washout devices. ERJ Open Res 2017;3(4).

85. Wagner PD, Saltzman HA, West JB. Measurement of continuous distributions of ventilation-perfusion ratios: theory. J Appl Physiol 1974;36(5):588-599.

86. Hopkins SR, Wagner, P.D. Review of the MIGET Literature, The Multiple Inert Gas Elimination Technique (MIGET) pp 145-233; 2017.

87. Wagner PD, Dantzker DR, Dueck R, et al. Ventilation-perfusion inequality in chronic obstructive pulmonary disease. J Clin Invest 1977;59(2):203-216.

88. West JB. Causes of and compensations for hypoxemia and hypercapnia. Compr Physiol 2011;1(3):1541-1553.

89. Rodriguez-Roisin R, Drakulovic M, Rodriguez DA, et al. Ventilation-perfusion imbalance and chronic obstructive pulmonary disease staging severity. J Appl Physiol (1985) 2009;106(6):1902-1908.

90. Wagner PD, Dantzker DR, Iacovoni VE, et al. Ventilation-perfusion inequality in asymptomatic asthma. Am Rev Respir Dis 1978;118(3):511-524.

91. Lagerstrand L, Bylin G, Hedenstierna G, et al. Relationships among gas exchange, spirometry and symptoms in asthma. Eur J Med 1992;1(3):145-152.

92. Roca J, Ramis L, Rodriguez-Roisin R, et al. Serial relationships between ventilation-perfusion inequality and spirometry in acute severe asthma requiring hospitalization. Am Rev Respir Dis 1988;137(5):1055-1061.

93. Bergin $\mathrm{C}$, Muller $\mathrm{N}$, Nichols DM, et al. The diagnosis of emphysema. A computed tomographic-pathologic correlation. Am Rev Respir Dis 1986;133(4):541546. 
94. de Jong PA, Muller NL, Pare PD, et al. Computed tomographic imaging of the airways: relationship to structure and function. Eur Respir J 2005;26(1):140-152.

95. Hammond E, Sloan C, Newell JD, Jr., et al. Comparison of low- and ultralowdose computed tomography protocols for quantitative lung and airway assessment. Med Phys 2017;44(9):4747-4757.

96. Matin TN, Rahman N, Nickol AH, et al. Chronic Obstructive Pulmonary Disease: Lobar Analysis with Hyperpolarized (129)Xe MR Imaging. Radiology 2017;282(3):857-868.

97. Lynch DA, Al-Qaisi MA. Quantitative computed tomography in chronic obstructive pulmonary disease. J Thorac Imaging 2013;28(5):284-290.

98. Lynch DA, Moore CM, Wilson C, et al. CT-based Visual Classification of Emphysema: Association with Mortality in the COPDGene Study. Radiology 2018:172294.

99. Bhatt SP, Soler X, Wang X, et al. Association between Functional Small Airway Disease and FEV1 Decline in Chronic Obstructive Pulmonary Disease. Am J Respir Crit Care Med 2016;194(2):178-184.

100. Boueiz A, Chang Y, Cho MH, et al. Lobar Emphysema Distribution Is Associated With 5-Year Radiological Disease Progression. Chest 2018;153(1):65-76.

101. Fernandes L, Fernandes Y, Mesquita AM. Quantitative computed tomography imaging in chronic obstructive pulmonary disease. Lung India 2016;33(6):646-652.

102. Harris RS, ,Venegas, J.G, Wongviriyawong, C., Winkler, T., Kone, M., Musch, G., Vidal Melo, M.F., de Prost, N., Hamilos, D.L., Afshar, R., Cho, J., Luster, A.D., Medoff, B.D. 18F-FDG uptake rate is a biomarker of eosinophilic inflammation and airway response in asthma. J Nucl Med 2014;52(11):1713-1720.

103. Ebner L, Kammerman J, Driehuys B, et al. The role of hyperpolarized (129)xenon in MR imaging of pulmonary function. Eur J Radiol 2017;86:343-352.

104. Mugler JP, 3rd, Altes TA. Hyperpolarized 129Xe MRI of the human lung. J Magn Reson Imaging 2013;37(2):313-331.

105. Thomen RP, Sheshadri A, Quirk JD, et al. Regional ventilation changes in severe asthma after bronchial thermoplasty with (3)He MR imaging and CT. Radiology 2015;274(1):250-259.

106. Svenningsen S, Eddy RL, Lim HF, et al. Sputum Eosinophilia and Magnetic Resonance Imaging Ventilation Heterogeneity in Severe Asthma. Am J Respir Crit Care Med 2018;197(7):876-884.

107. Marshall H, Horsley A, Taylor CJ, et al. Detection of early subclinical lung disease in children with cystic fibrosis by lung ventilation imaging with hyperpolarised gas MRI. Thorax 2017;72(8):760-762.

108. Kirby M, Svenningsen S, Owrangi A, et al. Hyperpolarized 3He and 129Xe MR imaging in healthy volunteers and patients with chronic obstructive pulmonary disease. Radiology 2012;265(2):600-610.

109. Sa RC, Henderson AC, Simonson T, et al. Measurement of the distribution of ventilation-perfusion ratios in the human lung with proton MRI: comparison with the multiple inert-gas elimination technique. J Appl Physiol (1985) 2017;123(1):136-146.

110. Ciaffoni L, O'Neill DP, Couper JH, et al. In-airway molecular flow sensing: A new technology for continuous, noninvasive monitoring of oxygen consumption in critical care. Sci Adv 2016;2(8):e1600560.

111. Mountain JE, Santer P, O'Neill DP, et al. Potential for noninvasive assessment of lung inhomogeneity using highly precise, highly time-resolved measurements of gas exchange. J Appl Physiol (1985) 2018;124(3):615-631. 
112. Smith N, Talbot N, Ritchie G, et al. Measuring lung inhomogeneity in asthma using a novel non-invasive technique. European Respiratory Journal 2018;52(suppl 62):PA3889. 
$\frac{7}{00}$

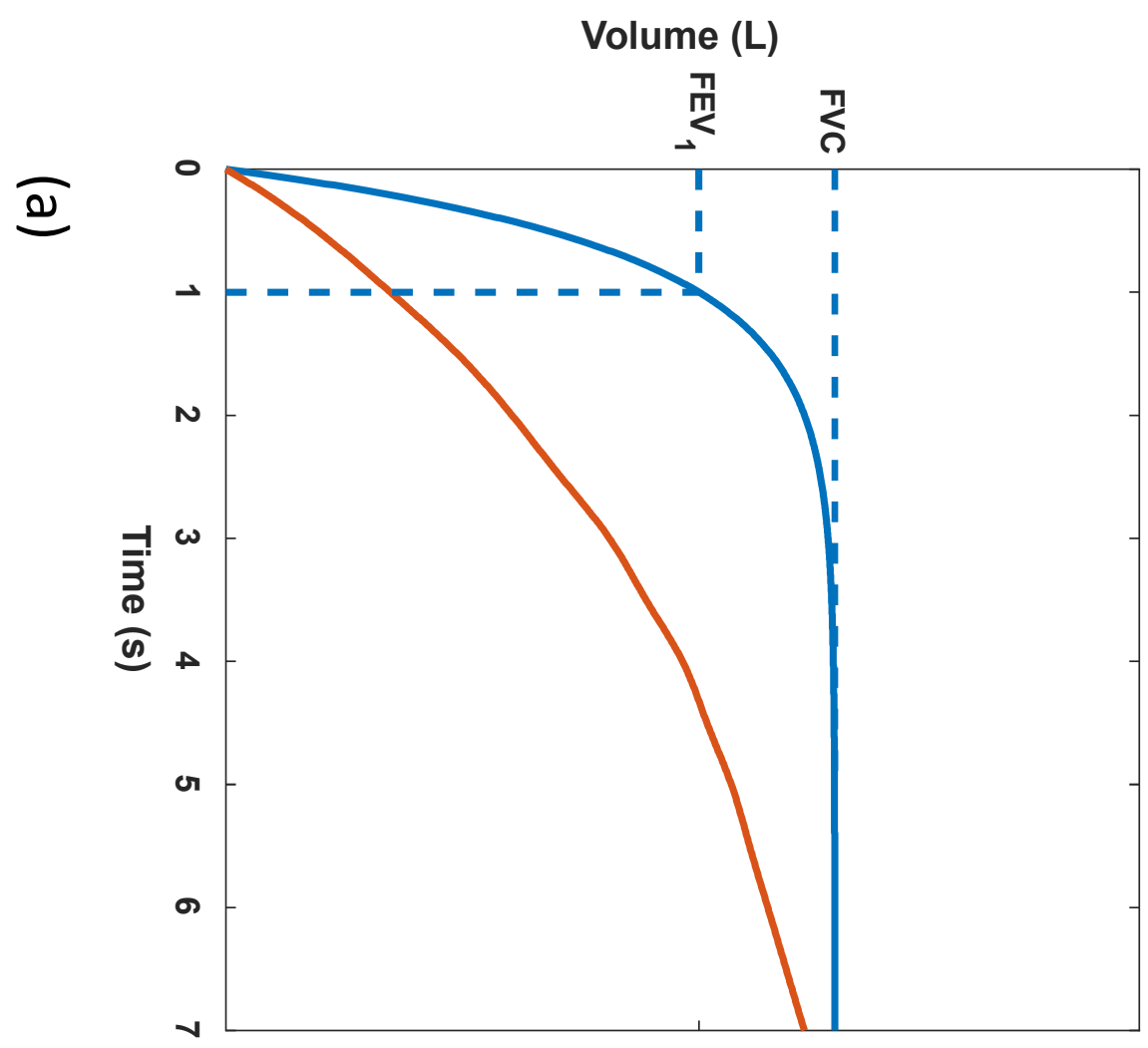

Flow (L/s)

$\widetilde{\sigma}$

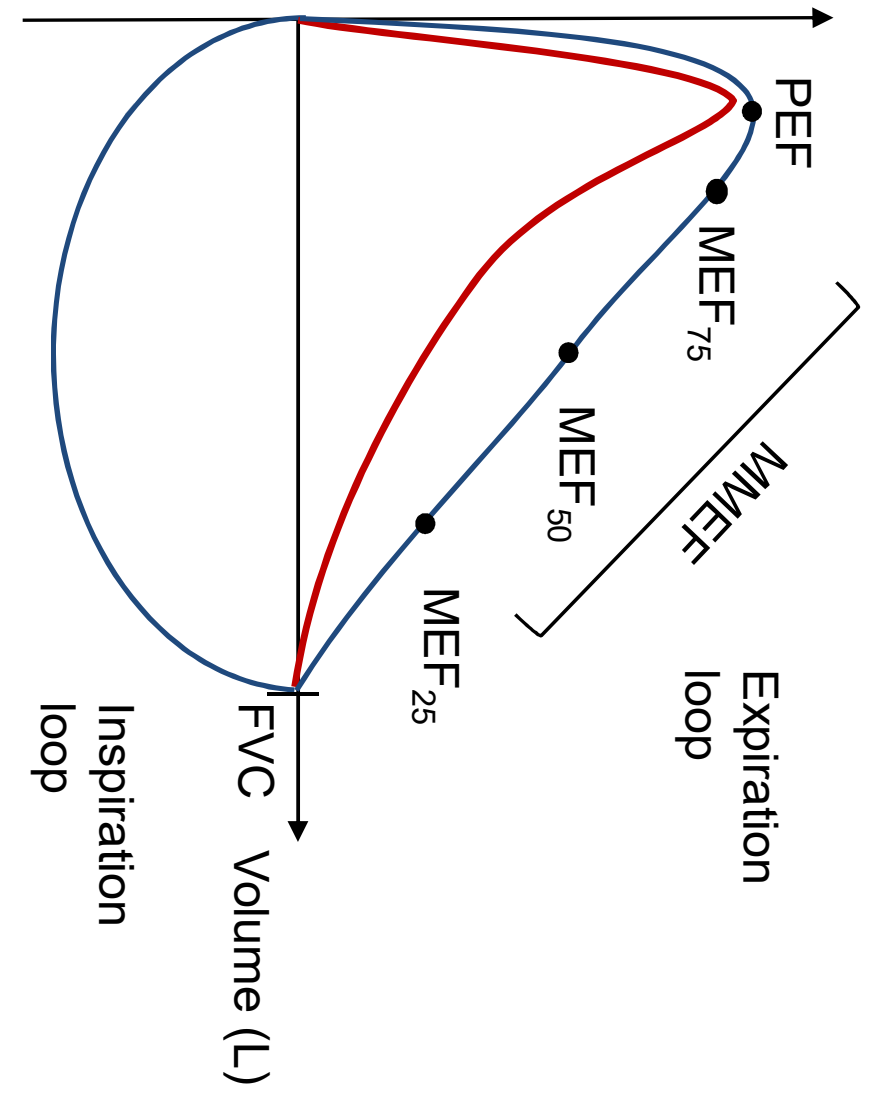


${ }_{N}^{\frac{T}{00}}$

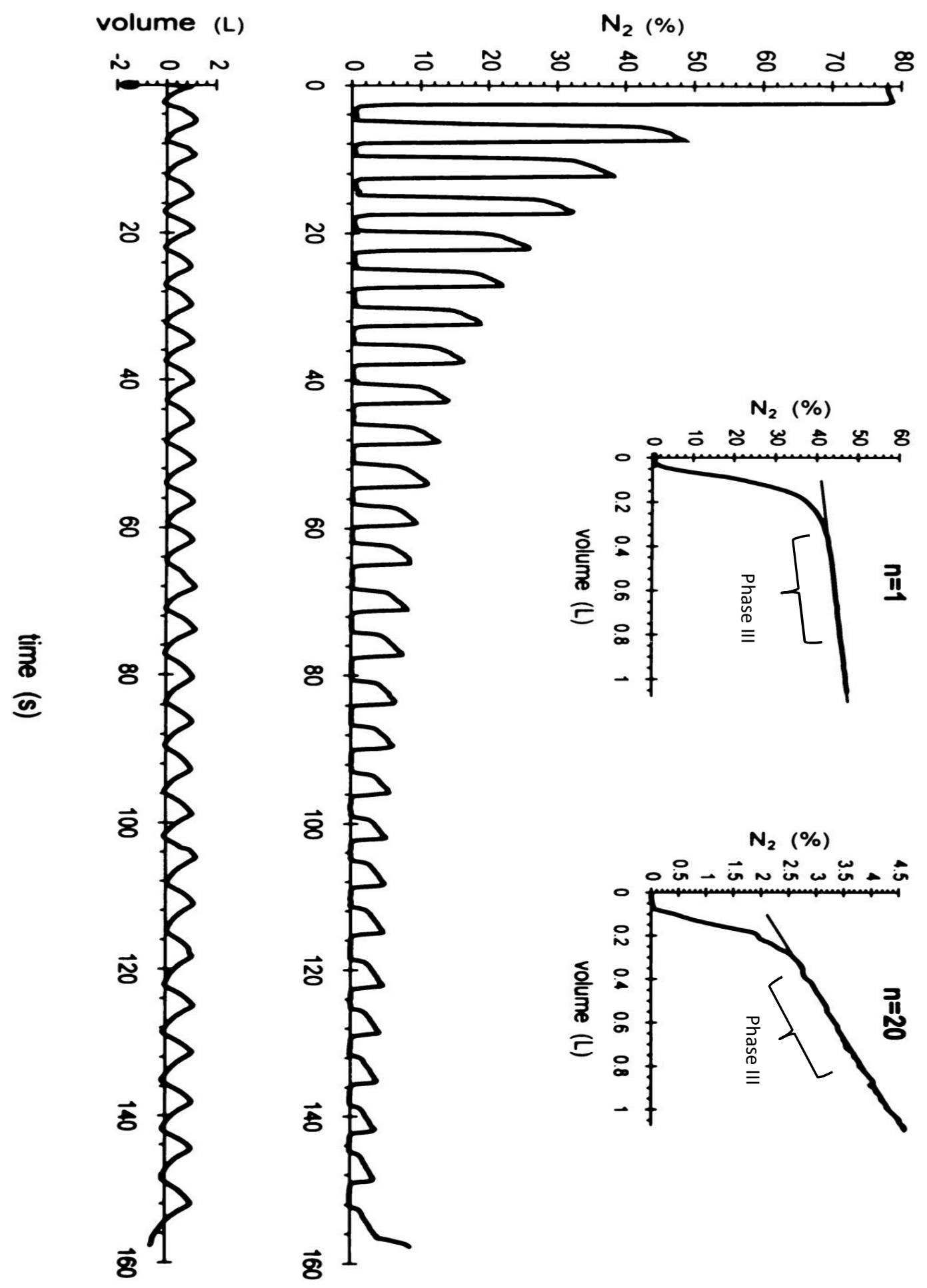


$\frac{7}{09}$
$\frac{0}{D}$
$\omega$
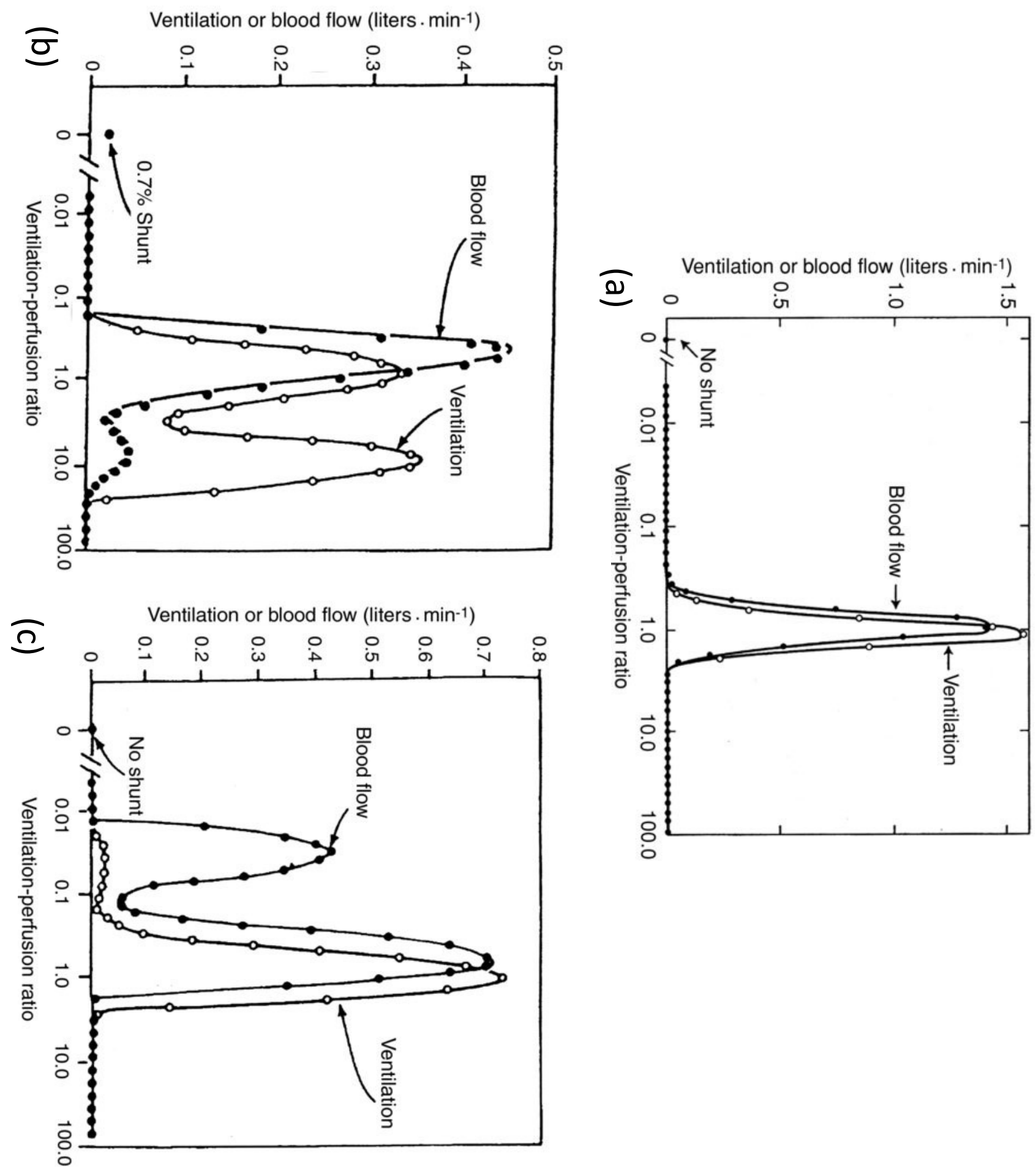
员

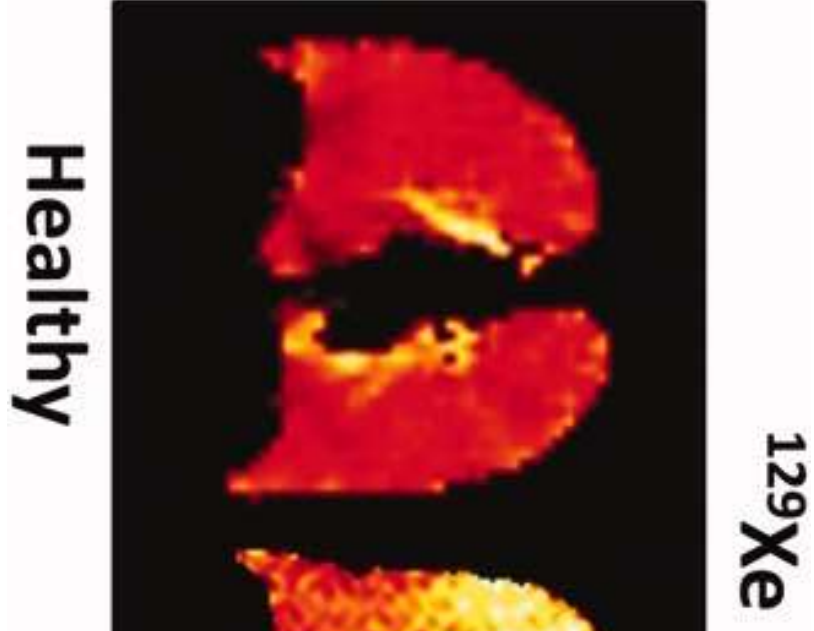

웜
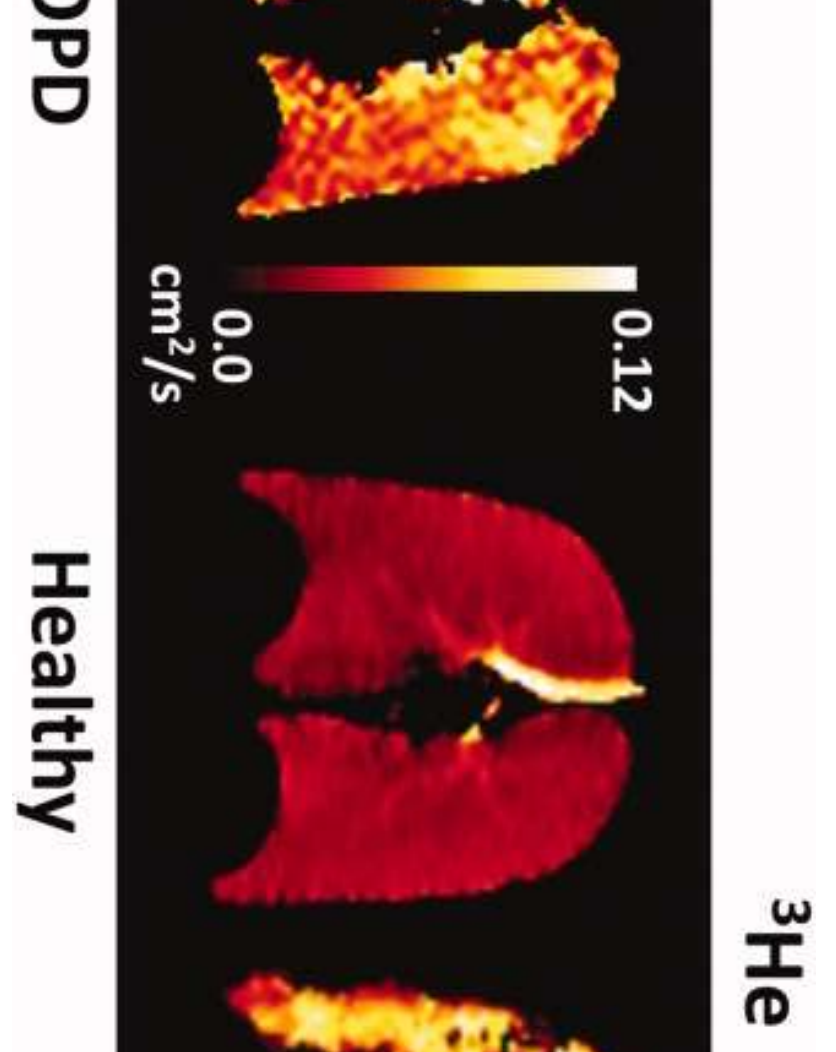

음

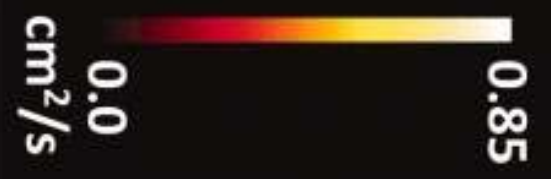


$\frac{T}{0 ! 9}$
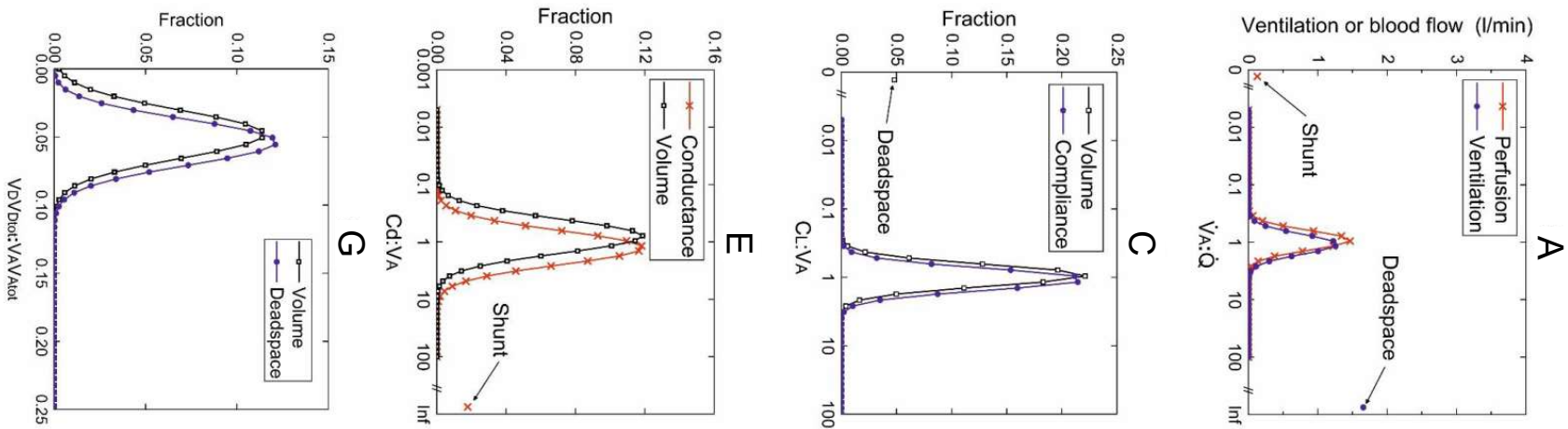

$\frac{I}{10}$
$\frac{0}{7}$
$\frac{1}{2}$
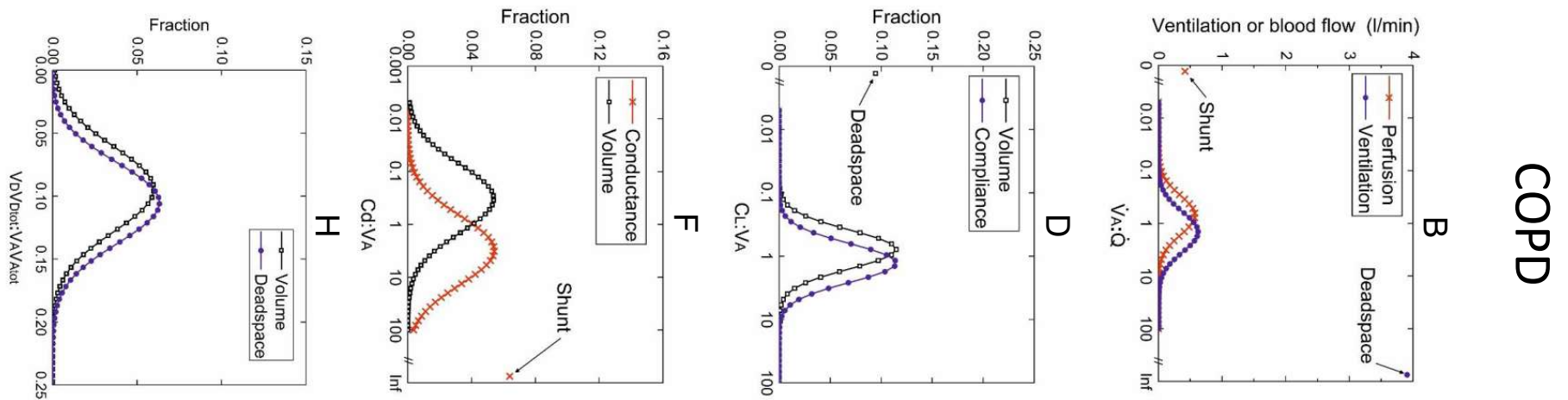